\title{
Visual Observation of Nucleate Boiling and Sliding Phenomena of Boiling Bubbles on a Horizontal Tube Heater
}

\author{
Jae Soon Kim, Yu-Na Kim, Goon-Cherl Park, Hyoung Kyu Cho* \\ Department of Nuclear Engineering, Seoul National University \\ Daehak-dong, Gwanak-gu, Seoul, 151-742, Republic of Korea \\ godot@snu.ac.kr, rladbsk0828@snu.ac.kr, parkgc@snu.ac.kr, chohk@snu.ac.kr*
}

\begin{abstract}
In the estimation of the mechanistic boiling heat transfer named wall heat flux partitioning model or RPI model, detail bubble behavior significantly influences the results. This mechanistic model is believed to have advantage from reflecting phenomena more correctly and be applicable to wider range of geometric and conditions by its constituting principle. Consequently, visualizing the phenomena over wide range of heating conditions around the bubble is important. But in conventional experimental approaches, there are some limitations. At high heat flux that exceeding the ONB(Onset-of-Nucleate Boiling) heat flux, visual observation becomes restricted by a number of bubbles are generated on a heater surface. In this study, we newly designed a heater based on the Flexible Printed Circuit Board (FPCB) for a better visualization of the nucleate boiling and sliding phenomena of boiling bubbles. It has narrow heating widths and it make possible to local heating of the surrounding fluids and locally generates bubble without any visual interference along the axial direction. Furthermore, a measurement technique was established in the interest of reconstruct the boiling bubbles and quantify their behavior based on an image processing. Two synchronized high-speed cameras were arranged at an angle of $90^{\circ}$ with each other to observe the bubble nature precisely. The bubble motion is simultaneously recorded in axial and side directions through these cameras from their birth to lift-off. Using the measurement technique, important parameters of the nucleate boiling are measured including bubble departure frequency, and bubble volume.
\end{abstract}

Keywords: Nucleate Boiling, Flexible Heater, Image Processing, Heat Partionoing, Bubble Sliding

\section{Introduction}

In nuclear reactor thermal-hydraulic safety analysis, detailed understanding of a boiling heat transfer is of great importance. Especially, in the mechanistic boiling heat transfer model named wall heat flux partitioning model or RPI model[1], detail bubble behavior significantly influences the estimation of the boiling heat transfer. Since this mechanistic model is footing on its nature, it is believed to have advantage from reflecting phenomena more correctly and be applicable to wider range of geometric and surrounding conditions. In this model, boiling heat transfer mechanism consists of bubble behaviors. Therefore, it is important to visualize the phenomena around the bubble precisely over wide range of heating conditions. In this study, we newly designed a heater based on the Flexible Printed Circuit Board (FPCB) for a better visualization of the bubble behavior. Subsequently, a measurement technique was established in order to reconstruct the boiling bubbles and quantify their behavior based on an image processing. Using the measurement technique, important parameters of the nucleate boiling are analyzed. This paper introduces the experimental setup, the digital image processing technique, and visualization results.

\section{Experiment}

The object of this experiment is to observe the boiling bubble on the horizontal tube heater because there are not much experimental data on horizontal tube geometry which can significant contribution on the heat transfer. Thus, the present experiment aimed to provide experimental data for the better understanding of the bubble motion and for the improvement of the bubble behavior models. To achieve these goal, we build experiment loop and designed special heater. Detailed description of the experiments will be introduced as follows. 


\subsection{Experimental Loop}

In this experiment, flow conditions like inlet pressure, temperature and mass flow can be controlled to observe the parameters in various thermal-hydraulic conditions. Prototype of this experiment, are targeting the surrounding conditions the Passive Auxiliary Feedwater System (PAFS) heat exchanger as normal operation[2]. Fig. 1. shows experimental apparatus and test-section, respectively. A test section is made to visualize the vapor bubbles on the surface of the heater. Also there are pre-heater and heat exchanger for heating inlet fluid and to avoid pump cavitation damage respectively. For de-aeration Re-flux condenser

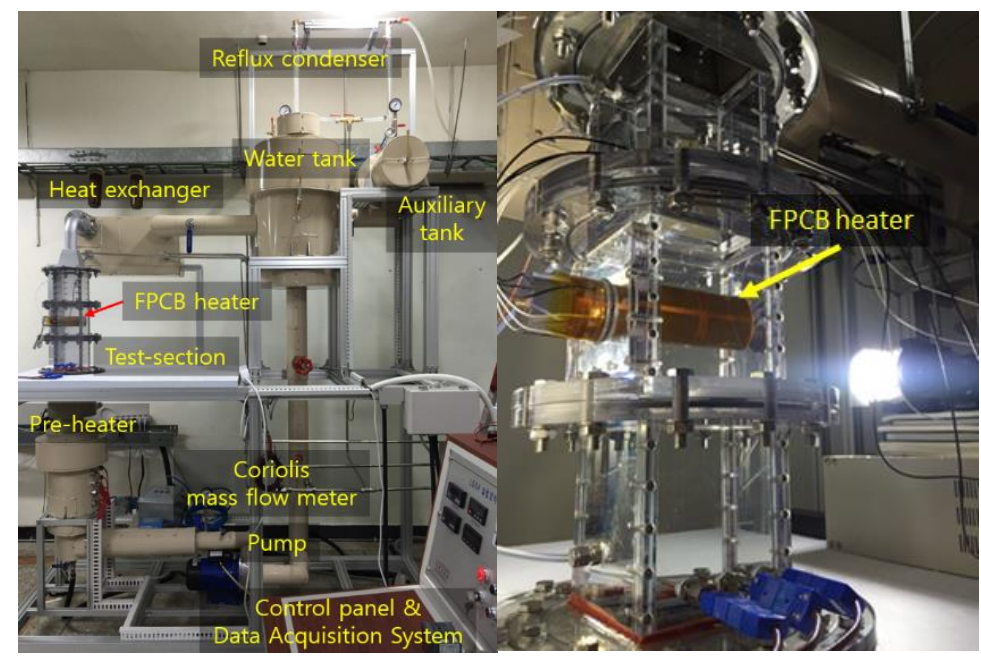

Fig. 1: Experimental apparatus.

\subsection{Horizontal Tube Heater Fabricated with Flexible Printed Circuit Board}

In the mechanistic boiling heat transfer model, parameters of an individual bubble are important to understanding the boiling phenomena. Therefore, understanding these parameters is important, but a conventional cartridge heater has problems in visualization as the number of bubble increases. As shown in Fig. 2 (a), the axial view parallel to the horizontal tune is interrupted by overlapped bubbles. Therefore, in this study, a heater fabricated with Flexible Printed Circuit Board (FPCB) was devised. It has $1 \mathrm{~mm}$ heating width to generate bubbles along a narrow region without interference by other bubbles along the observation direction (Fig. 2 (b)). Fig. 2 (c) shows the picture of the heater. Cylindrical polycarbonate tube not only supports the flexible board, also insulates heater inside. If voltage is imposed to the heater, narrow width has larger electrical resistance than the other region. Consequently, the area is locally heated and boiling occurs on the narrow band. With this new concept of the present heater, visualization became feasible without interference by overlapped bubbles.

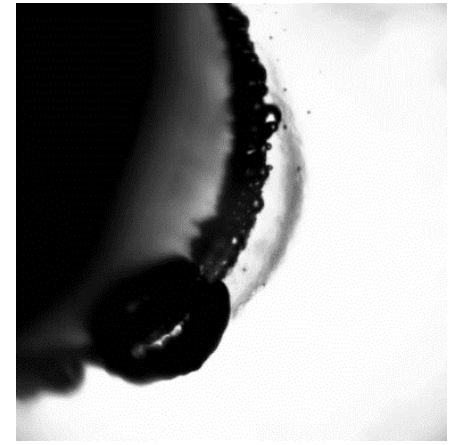

(a) axial view of cartridge heater

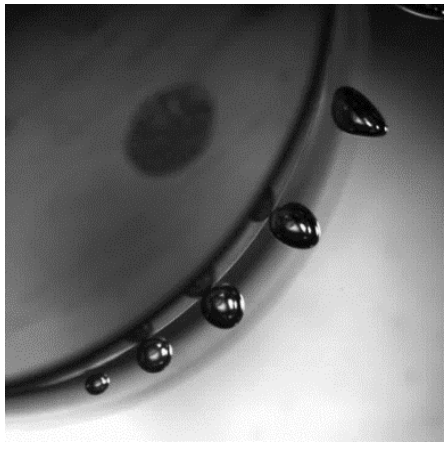

(b) axial view of film heater

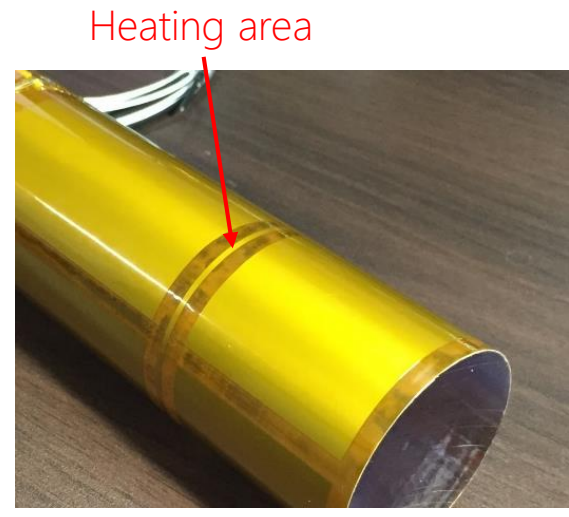

(c) film heater

Fig. 2: Axial view and film heater. 


\subsection{Measurement Instrumentation}

This study measures the liquid temperatures, pressures and flow rates during the experiment. And the bubble parameters were measured using two synchronized high-speed cameras. After experiment, recorded images are postprocessed through digital image processing technique.

\subsection{3-D Reconstruction}

Two images of a bubble from the axial and side views had phase separation and 3-D reconstruction procedures to measure the volume and bubble behaviors. For the 3-D reconstruction, a reconstruction method proposed by Kim et al.[3] was applied. This method accumulates extracted solids of which cross section is obtained from the combination of the both images. And the bubble volume prediction error of these method has less than $1 \%$ uncertainty.

\section{Results and Discussions}

\subsection{Experiment condition}

The bubble behavior was investigated with various wall heat flux and inlet liquid velocity. The water temperature is maintained at the saturation temperature and the pressure is maintained at nearly atmospheric pressure. The bulk velocity of the experiment ranged from $15 \mathrm{~mm} / \mathrm{s}$ to $28 \mathrm{~mm} / \mathrm{s}$ and wall heat flux varied from $67 \mathrm{~kW} / \mathrm{m}^{2}$ to $129 \mathrm{~kW} / \mathrm{m}^{2}$ which are in the nucleate boiling regime on boiling curves. In the present paper, the bubble motions generated at two different angles of the nucleation site, $45^{\circ}$ and $90^{\circ}$ from the bottom of the heater.

\subsection{General phenomena}

Figure 3(a) is the visual observation result of the bubble life-cycle from its incipience to the lift-off from the heating surface when the bubble is generated at $45^{\circ}$ nucleation site angle. Bubbles are periodically generated at a local point called nucleation site. The bubble starts to grow until its volume becomes large enough to depart from heating surface. Then, the bubble starts to move from the nucleation site and slides along the heating surface in a very short distance. Then, the bubble lifts off from the heating surface and the first lift-off occurs. After the first lift-off, bubble receives the buoyancy force and it moves up and re-attaches on the heating surface. In sequence, slides on the curved heating surface and its size increases gradually at the same time. If the bubble size is sufficiently large during its sliding, the lifts off from the surface again, the second lift-off occurs. When the bubble is generated at $90^{\circ}$ nucleation site angle, there is no sliding effect. All these phenomena can be described with the force balance acting on a bubble[4].

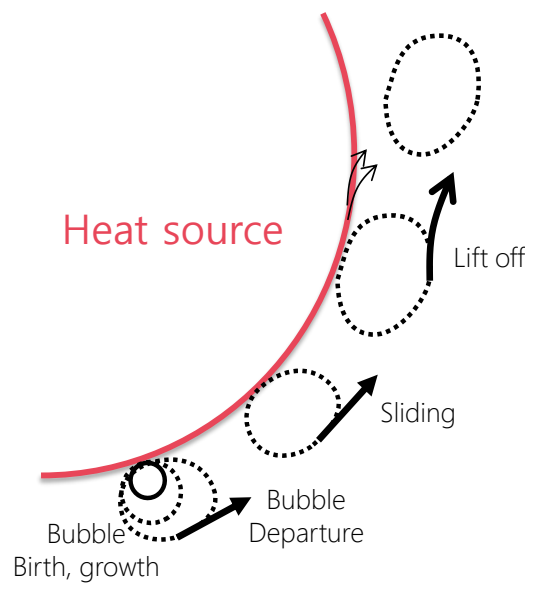

(a) Schematic of bubble life-cycle at $45^{\circ}$

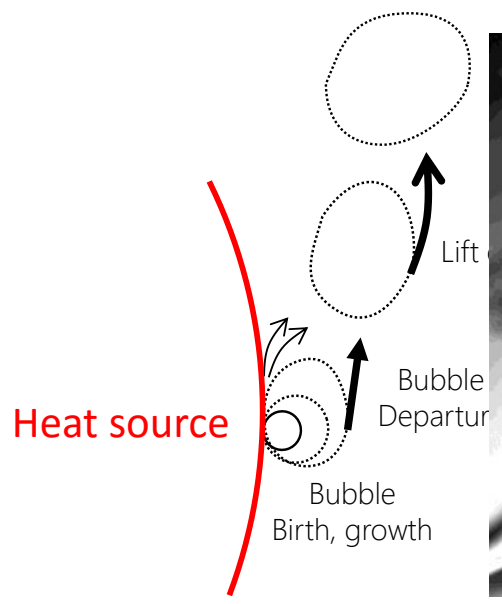

(b) bubble life-cycle at $90^{\circ}$

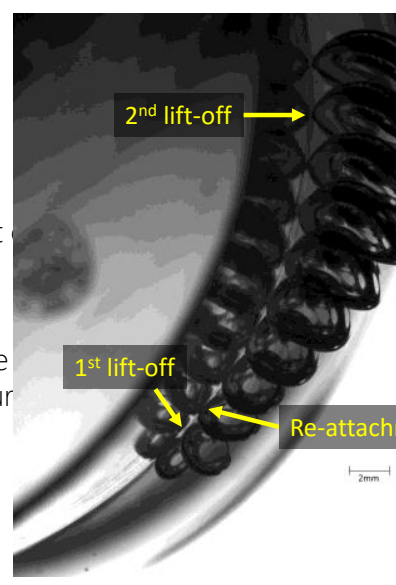

(b) bubble life-cycle at $45^{\circ}$

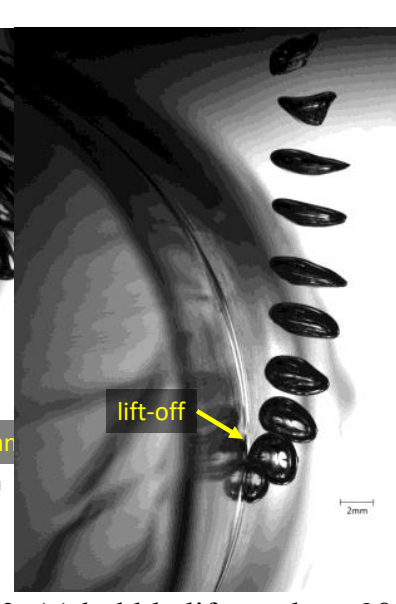

(c) bubble life-cycle at $90^{\circ}$

Fig. 3: Bubble sliding phenomena.

\subsection{Effect of heat flux}

As the heat flux increases, bubbles are more frequently generated and more bubbles are observed in the same visualization frame. Thus, the measured bubble frequency shows increasing trend in general with increasing heat flux as 
shown in Fig. 4. If the heat flux increases, the bubble growth rate is raised and the contact pressure force is enlarged. This increased contact pressure force results in more frequent lift-off of the bubbles with smaller diameters.

In the case of the $45^{\circ}$ nucleation site angle, the bubble slides over the heater surface after the reattachment and the bubble size increases. The increasing slope of the sliding bubble volume, however, decreases with the heat flux as presented in Fig. 5. This can be attributed to the establishment of the superheated layer and the agitation effect of the sliding bubble. Once a bubble slides up, it disturbs the superheated liquid layer made on the heating area and surface quenching occurs. Since sliding bubbles disturb the superheated liquid layer more frequently, the growth rate of the sliding bubble is reduced with the bubble frequency. A similar trend was observed in the case of the $90^{\circ}$ nucleation site angle. The larger the heat flux is, the smaller bubble departure diameter was obtained.

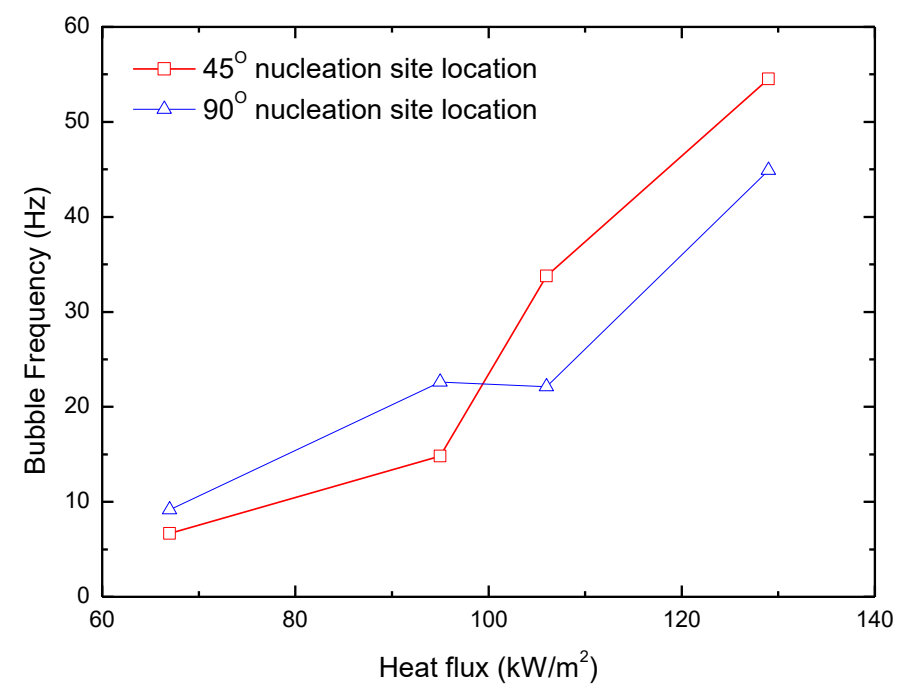

Fig. 4: Bubble frequency vs. heat flux $\left(v_{l}=\mathbf{2 2} \mathbf{m m} / \mathrm{s}\right)$.

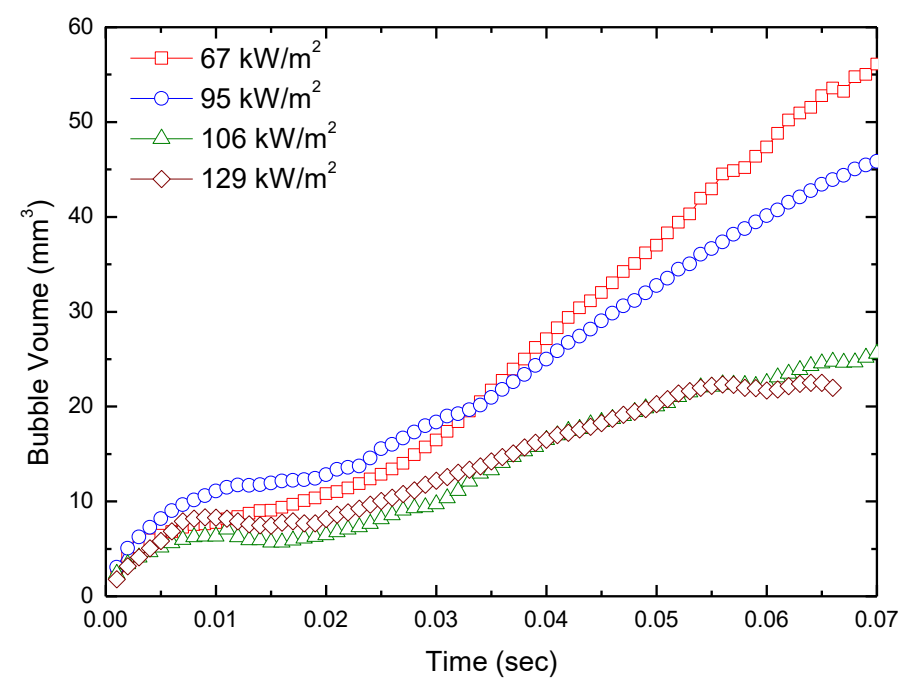

(a) $45^{\circ}$ nucleation location

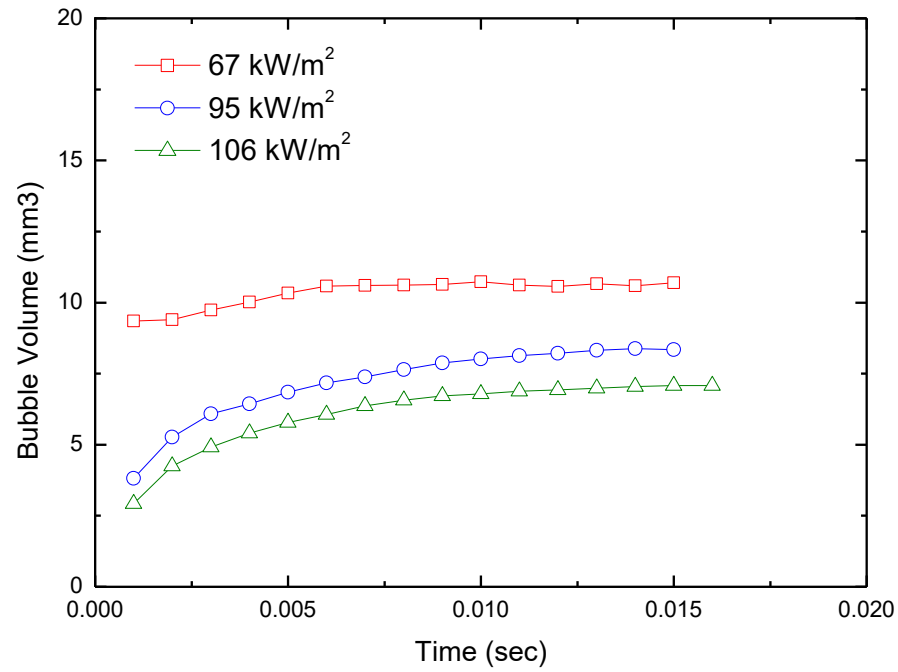

(b) $90^{\circ}$ nucleation location

Fig. 5: Measured bubble volume transient: heat flux effect $\left(v_{l}=22 \mathrm{~mm} / \mathrm{s}\right)$.

\subsection{Effect of velocity}

Higher liquid velocity transfers more drag force to the sliding bubble if the liquid velocity is faster than bubble velocity and less drag force in the other cases. Therefore, the bubble velocity increases with the liquid velocity. However, 
the bubble volume transient was not significantly affected by the liquid inlet velocity as shown in Fig. 6 . This is caused by that the bubble slides longer with the higher liquid velocity than the lower case.

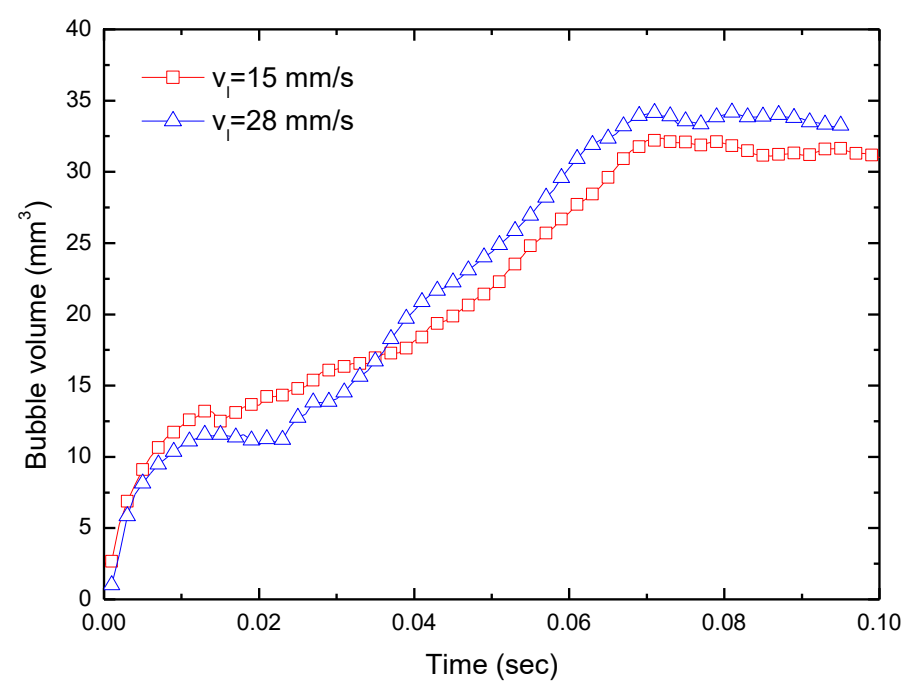

Fig. 6: Bubble velocity transient $\left(45^{\circ}\right.$ nucleation location, $\left.q^{\prime \prime}{ }_{l}=\mathbf{1 0 6} \mathbf{k W} / \mathbf{m}^{2}\right)$.

\section{Conclusion}

In this study, nucleate boiling and sliding phenomena observation experiment on horizontal tube is conducted. For this reason, heater is specially designed and also the stereoscopic image processing method is proposed. Through the techniques, various bubble behavior are measured such as bubble volume, and frequency. In the future, the experimental results will be used to verify bubble behavior and force balance models and improve the heat partitioning model for the horizontal tube heater.

\section{Acknowledgements}

This work was supported by the Nuclear Safety Research Program through the Korea Foundation Of Nuclear Safety(KoFONS), granted financial resource from the Nuclear Safety and Security Commission(NSSC), Republic of Korea. (No. 1305011)

\section{References}

[1] N. Kurul and M. Z. Podowski, "Multidimensional effects in forced convection subcooled boiling," Heat Transfer Conference, vol. 2, pp. 19-24, 1990.

[2] K. H. Kang et al., "Separate and integral effect tests for validation of cooling and operational performance of the APR+ Passive Auxiliary Feedwater System," Nuclear Engineering and Technology, vol. 44, no. 6, pp. 597-610, 2012.

[3] S. J. Kim and G. C. Park, "Interfacial heat transfer of condensing bubble in subcooled boiling flow at low pressure," International Journal of Heat Mass Transfer, vol. 54, pp. 2962-2974, 2011.

[4] J. F. Klausner, "Vapor bubble departure in forced convection boiling," Int.J.Heat Mass Transfer, vol. 36, no. 3, pp. 651-662, 1993. 